\title{
Biomechanical Signals Human-Computer Interface for Severe Motor Disabilities
}

\author{
Albano Carrera, Alonso A. Alonso, Ramón de la Rosa, Javier M. Aguiar \\ Laboratory of Electronics and Bioengineering, Department of Signal Theory, Communications and Telematics Engineering, Higher \\ Technical School of Telecommunications Engineering, Universidad de Valladolid, Valladolid, Spain \\ Email: albano.carrera@uva.es, alonso3@tel.uva.es, ramros@tel.uva.es, javagu@tel.uva.es
}

Received July 31, 2013; revised August 30, 2013; accepted September 20, 2013

Copyright (c) 2013 Albano Carrera et al. This is an open access article distributed under the Creative Commons Attribution License, which permits unrestricted use, distribution, and reproduction in any medium, provided the original work is properly cited.

\begin{abstract}
A system that allows computer interaction by disabled people with very low mobility and who cannot use the standard procedure based on keyboard and mouse is presented. The development device uses the patient's voluntary biomechanical signals, specifically, winks - which constitute an ability that generally remains in this kind of patients-, as interface to control the computer. A prototype based on robust and low-cost elements has been built and its performance has been validated through real trials by 16 users without previous training. The system can be optimized after a learning period in order to be adapted to every user. Also, good results were obtained in a subjective satisfaction survey that was completed by the users after carrying out the test trials.
\end{abstract}

Keywords: Man-Machine Systems; Electronics; Medical Rehabilitation; Independent Living; Biomedical Engineering

\section{Introduction}

The present paper is placed in the Rehabilitation Technologies (RT) field, in particular in the human-machine interfaces' development and its application to different systems with the aim of improving the quality of life and the personal autonomy of the disabled. Specifically, this work deals with the implementation of a human-computer interface for severe motor disabilities and those who cannot use the standard ways to control a computer, mouse and keyboard, due to their disability.

Currently, there are several papers focused on the field of RT and on the development of adapted interfaces for the interaction with different devices. These interfaces should meet a set of requirements, which can be found in the related scientific literature, to ensure the best performance [1]. Due to the great diversity of interfaces that can be developed, a preliminary study of adaptation to the application of interest is necessary. Thus, a revision of the related scientific literature has been carried out, and different kinds of interfaces have been found: voice recognition [2], video camera-based systems [3], bioelectric signals, such as the electromyogram (EMG) or the electrooculogram (EOG), while performing voluntary movements [4], electroencephalogram (EEG) signals [5], residual movement detection without utilising bioelectric signals [6], inertial sensors [7] and autonomous interaction after activation [8]. Previous analysis carried out by the research group [1] advises and justifies the use of interfaces based on residual voluntary movement detection without utilizing bioelectric signals for the tasks of command detection generated by users with severe motor disabilities.

Focusing our attention on this kind of human-machine interfaces, the residual voluntary movement detection without utilizing bioelectric signals, a few projects based on the registration of different gestures or corporal movements can be found: movements of the head [9], winks combined with movements of the head [10], movements of the tongue [11], sniffing [12] and posture changes [13]. Among the above mentioned options, wink detection has been considered the most adequate because this ability prevails in most of the severe motor disability cases and it is the gesture that interferes less with the normal daily activity of the subject.

The interfaces mentioned above have been utilised for the control of RT systems, such as electric wheelchairs [1,14], surveillance systems [1] and, also, personal computers [3]. In this paper, the interfaces employed to control personal computers are of special interest and there are articles that refer to interfaces that use movements of 
the head [9], movements of the tongue [11], inertial sensors [15], image recognition systems that detect movements of the head [3], eyes [16] or body movements [17], bioelectrical signals, such as EMG [18,19], and movements of the head combined with winks [10]. The aforementioned paper presents a system that employs two different remaining abilities: one of them is the movement of the head to control the mouse. This ability, generally, does not prevail in severely disabled patients and, notwithstanding, its use constitutes a greater impediment for a simultaneous daily activity, while the patient is using the computer.

This paper presents a computer control system, by means of a human-machine interface based on residual movements that do not require bioelectric signals. In order to improve the discussion, this paper has been divided into different sections. The following one presents the aims set in the research work. Section three introduces the implemented material for the parts of the system: interface, processing system and computer. Section four presents the results and, finally, Section five presents the conclusions we arrived at, after the development, implementation and trials performed with the system.

\section{Objectives}

The main objective of the device presented in this paper is the design and implementation of an augmentative communication system that would allow a computer control for severe motor disabilities. Essentially, this control consists in the handling of the mouse and the keyboard by means of an adapted human-machine interface, although the system includes additional functions that have been optimised, such as the adapted wheelchair driving training or the domotic control.

The human-machine interface to control the system is based on the detection of user-simple gestures, without utilising bioelectric signals. Thus, biomechanical signals would be used to command the device and, specifically, detecting the user's voluntary winks.

Very severe motor disabilities preserve certain move- ment abilities, that the patient can make use of. In most cases, disabled people can make facial gestures: random eye movements, blinks and winks. The fact that a system based on voluntary winks, in which closing the eyelid is not necessary, should allow the user of the system to keep on utilising other residual abilities in order to interact with the environment, such as sight, hearing or voice. This means that a RT system which does not interfere with the patient's usual activities is the purpose of our human-machine interface.

\section{Methods}

The global structure of the augmentative communication system is very simple (Figure 1), as shown in the diagram of the three different parts of the system. First of all, the set of adapted interfaces developed for computer control detecting voluntary winks. Secondly, the processing system in charge of discrimination orders by the users. Finally, the functions of the computer where the developed software runs.

\subsection{Adapted Interface}

The first part of the equipment is the adapted interface. This interface, as explained above, is responsible for the reception of the user's voluntary winks. For the detection of these biomechanical signals, the implementation of different interfaces has been developed by the research group [1]. The function of the interface chosen is based on the light reflexion or not, according to a user's voluntary gesture. This task is carried out with a CNY70 device, which has a light emitter and an infrared phototransistor. The led light emitted reflects on a surface and depending on the nature of the surface the light will find a reflection or not. In this case, a bicolour adhesive tape (black and white) on the user's orbicularis oculi muscle skin, on the edge of the eye, is used to achieve a correct performance (Figure 2). The adhesive tape moves when a voluntary wink is performed, and the consequent movement causes a colour change and, hence, a change on the output of the device.

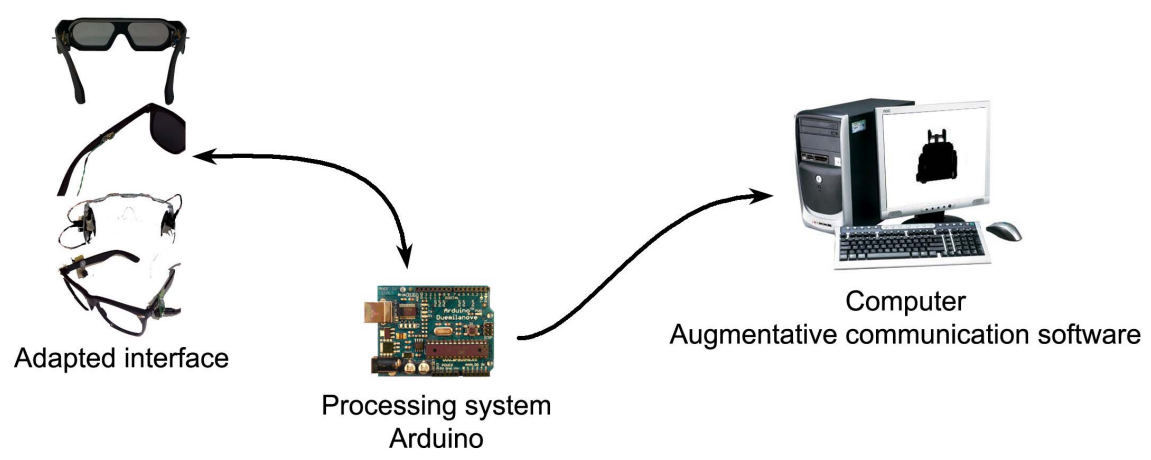

Figure 1. Augmentative communication system block diagram. 
With the aim of getting the best comfort and user's adaptability, the wink detectors were implemented on conventional glasses and mechanical adjusting elements to improve the performance were included. The humanmachine interface final aspect is shown in Figure 3.

\subsection{Processing System}

As the system needs a low computational load, as it only has two logic sensors with binary output, a microcontroller as process device was chosen. Specifically, an Arduino hardware platform, which implements a microcontroller and all the additional elements in a commercial board, was utilised.

Starting with this process device and its free development environment, a software algorithm for the detection of all wink gestures was developed. The gestures to be detected are: left wink, right wink, eyes closing wink, consecutive left-right winks and consecutive right-left winks, an array of possibilities.

The implemented program flowchart (Figure 4) employs the microcontroller interrupt functions in order to get the best efficiency in command detection. In order to avoid fake detections a duration wink timer threshold is incorporated.

Figure $\mathbf{4}$ flowchart omits the implicit thresholds in the detection functions. After the detection of one of the five patterns defined by the winks combination, the corresponding control command is sent to the computer. These commands are shown at the bottom of the flowchart ("left", "left + right", "right + left", "right" and "both eyes" action blocks) on a generic form, and different actions can be assigned depending on the application or the system handled.

\subsection{Computer. Augmentative Communication Software}

The communication between the personal computer and the wink detection adapted interface requires the implementation of specific software that will also be entrusted the running of the task regarding the augmentative communication software.

As explained above, this software allows mouse and keyboard control on a personal computer, it also includes other modules such as the control of virtual adapted electric wheelchairs or domotics devices.

With the aim of getting a scalable design, the software implementation has been made dividing it in different modules. Thus, progressively, new modules can be added, i.e., new system functionalities without the modification

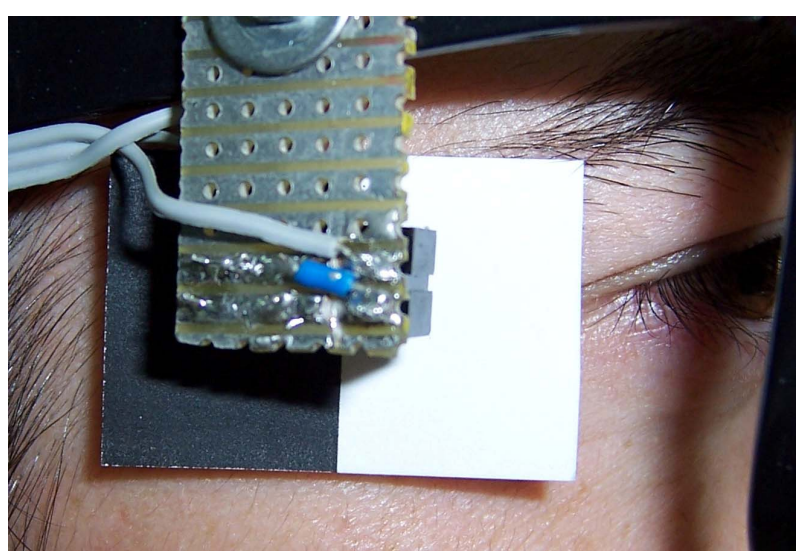

Figure 2. Detail of the adhesive tape used for correct performance of the light reflection sensors on the user's skin.

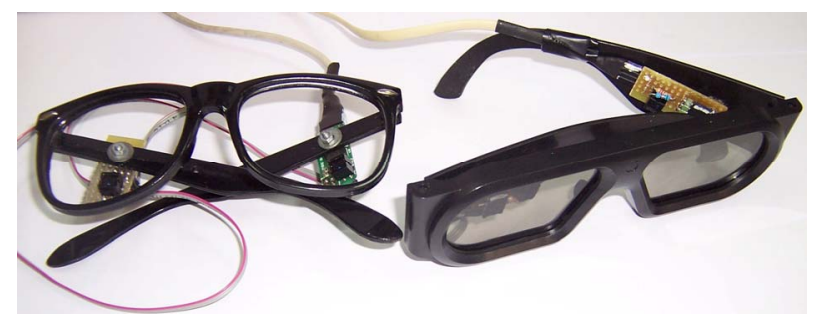

Figure 3. Human-machine interface of biomechanical signals for the detection of voluntary winks.

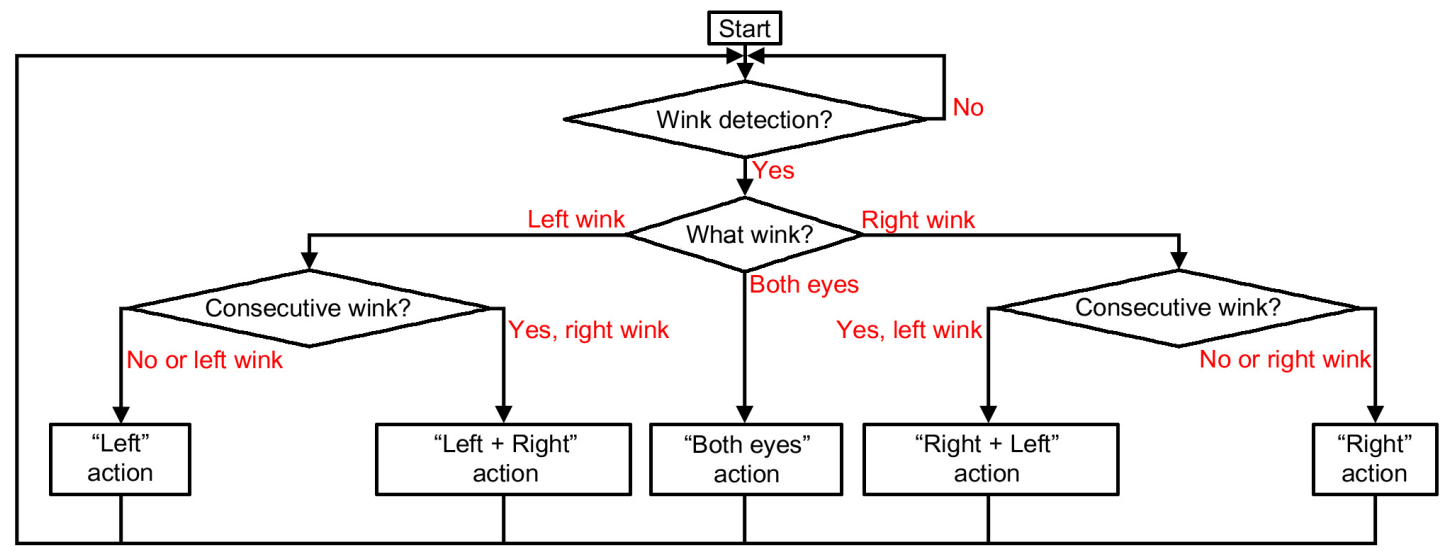

Figure 4. Algorithm flowchart implemented for the Arduino processing system. This flowchart shows the recognition of the five basic wink patterns that corresponded to control commands. 
of the existing ones; additionally, a user database was created to improve the user's interaction and save the personal configuration which can define for example, the time between two consecutive winks.

\subsubsection{Mouse Module}

The software mouse module controls the mouse on the computer screen with a full interaction with the computer as with a standard mouse. This means that a full movement on the screen is possible and also the left and right button clicks. Figure 5 shows the state diagram including possible operations.

Figure 5 shows the winks with a letter code: $\mathrm{L}$ is left wink and $\mathrm{R}$ is right wink, i.e., for the consecutive winks a similar codification is used: $\mathrm{L}+\mathrm{R}$ corresponds to left and right consecutive winks and $\mathrm{R}+\mathrm{L}$ to right and left consecutive winks. For the movement states, vertical or horizontal, once the cursor starts to move, it can be stopped winking and any of the Figure 5 states will remain active. An always visible help window was created to improve the user's control of the application.

\subsubsection{Keyboard Module}

The keyboard module, whose control commands are similar to the mouse module, allows a biomechanical signals virtual keyboard to be used by a disabled user. The implemented keyboard is optimised in order; the letters are ordered by a Spanish utilisation frequency, where the point of maximum frequency is the keyboard centre (Figure 6). The handling of the keyboard is similar to the state diagram shown in Figure 5 and the wink codification, with the exception that in this case the right clicks not exist. In order to facilitate the user interaction, as in the case of the mouse module, a brief summary with the possible actions is included.

This module incorporates different keyboards in order to include specific functionality groups, the same as in a standard keyboard that includes special keys. Thus, the left keys in Figure 6 keyboard: "Num/Esp”, "Bloq. May”,

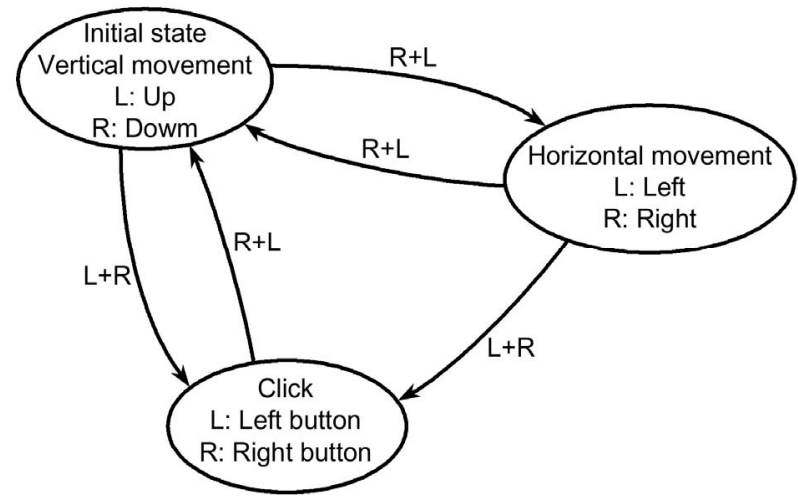

Figure 5. Function state diagram for the implemented mouse module.

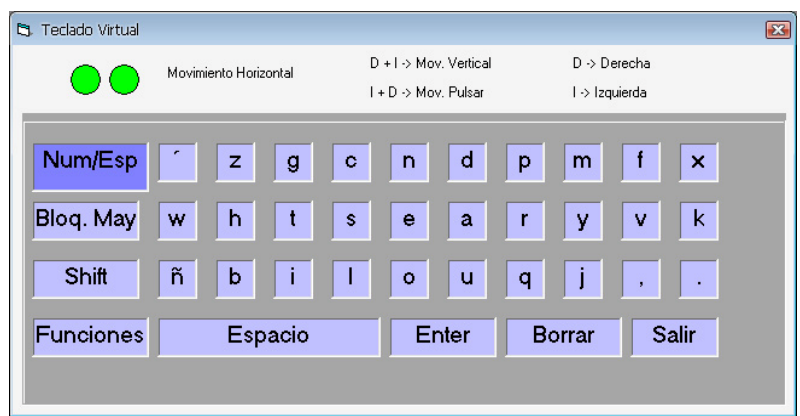

Figure 6. Virtual keyboard aspect with the letters ordered according to their Spanish utilisation frequency.

“Shift”, "Funciones” and “'”, give access to specific keyboards:

- Num/Esp keyboard. This keyboard allows the use of numbers and special characters. Once the required key has been clicked, the software returns automatically to the lower case letter keyboard (Figure 6).

- Capital letter keyboard. The access to this keyboard is done by "Bloq. May" key or by "Shift" with one touch only, and it can include capital letters.

- Functions keyboard. This keyboard is for special functions and it allows the performing of actions that normally are done with a keyboard and require the combination of different keys, such as copy, paste, cut, tab, window change, select and displacement arrows.

- Acute accent keyboard. This keyboard allows the use of written accent vowels both capital and lower case letters, it can be accessed by the "'” key and after the click the software returns to Figure 6 keyboard.

Therefore, this keyboard module allows to write and use the standard keyboard facilities in any application by means of an adapted human-computer interface.

\subsubsection{Other Modules}

Additionally to the two modules presented above, mouse and keyboard, another two have been included that allow different operations.

The first module is a configuration module where interface operation and interaction trials can be performed (Figure 7). In this way, a user can verify if the orders are correctly detected and his/her skill regarding the system will be shown in a configuration window.

The second one is an adapted wheelchair training application, which allows for trials to be carried out with a virtual wheelchair that simulates the movements of an adapted wheelchair in a specific environment (Figure 8). Like this, the wheelchair can move forwards, backwards or turn either left or right. With this module both training and improving of the adapted wheelchair driving tasks are expected and also the study of a possible purchase of a real wheelchair. 


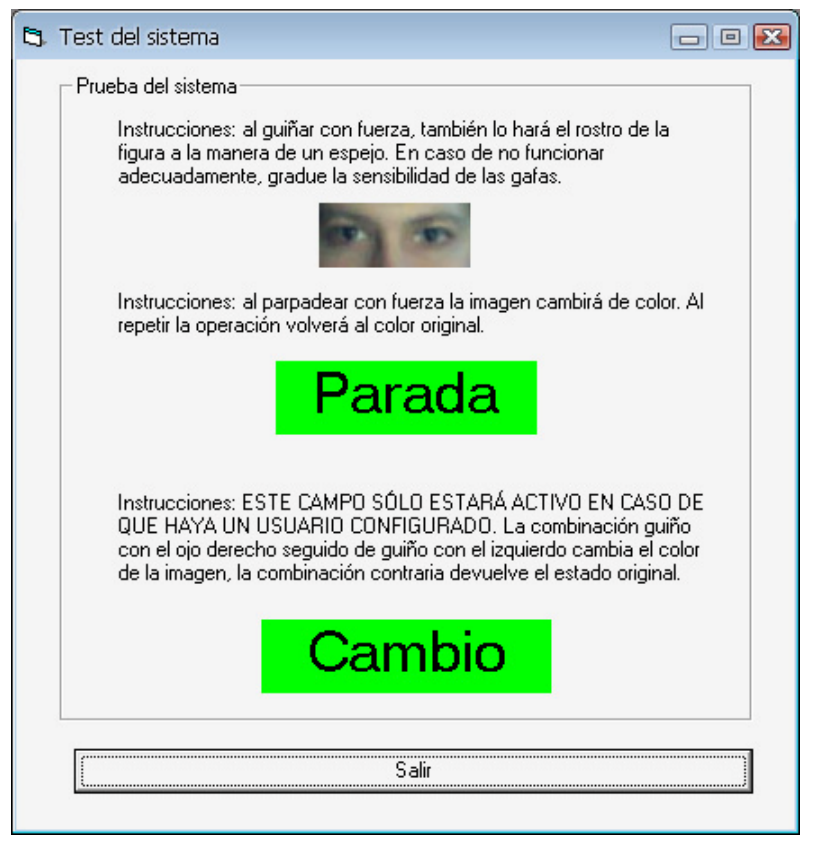

Figure 7. Aspect of the configuration and trial module.

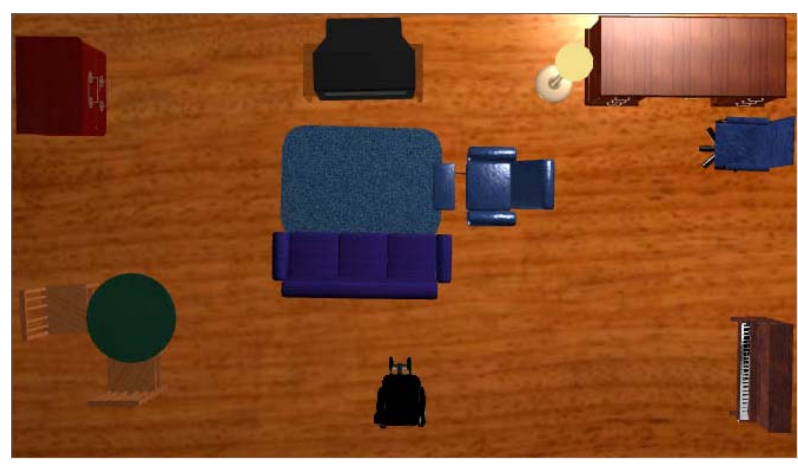

Figure 8. Aspect of the adapted wheelchair simulation module. In the picture, one of the implemented environments.

Also, another module, still in progress, has been included and it would allow tasks automation in a domotic environment.

\section{Results}

The assessment of the augmentative communication system developed takes into account both the prevailing abilities, such as voluntary winks, in severely motor disabled patients and control subjects. In most cases of interest this assumption is true, and the handling of this kind of gestural actions can be even much highly developed by some patients due to a compensation for other lost abilities. Therefore, trials were carried out on 16 healthy subjects, without the handicaps involving a loss of the validity of the results.

For these trials a specific protocol was designed. This protocol consists in the writing of a preset phrase and clocking the time taken to complete it. Then the control subject does a survey concerning the adapted interface used. Specifically, the writing trial is to complete two spanish phrases: "Hola mundo. Esto es una prueba.". This text, in spite of being very simple, involves clicking on 33 different keys (letters, capital letters, spaces and punctuation), so it is very appropriate to test the userfriendliness of the system.

The trials evaluate objective results such as the time to each subject to write the phrases, without any previous training. The average time to write the text was 8 minutes and 42 seconds. The average time to access and click each key was 15.8 seconds. Certainly, if the subjects train with the system and the interface, the handling of the equipment would improve, due to learning and familiarisation. This learning is reflected on some subjects.

The number of voluntary winks needed to complete the preset phrases was 363, taking into account clicking and column and row choices. The number of winks can be increased if errors are produced when the user is choosing the key or movement. In addition, as the number of gestures is high, the use of the system may cause fatigue; this effect can be supplemented with training or the incorporation of technical steps for writing improvement such as a prediction dictionary.

As explained previously, the subjects filled in a survey where they assessed various parameters of the wink detection interface. The evaluation scale employed from 0 , the worst score, to 5, the best (Table 1).

The results obtained in the survey reflect user's satisfaction with the system. Control subjects greatly appreciated the convenience and the easy use of the interface and the orders code. In the specific case of the system delay, a slightly lower rating was given because orders that require two consecutive winks for execution accumulated delay. This problem can be solved through training and customised software to shorten waiting times to include a second wink.

\section{Discussion}

An augmentative communication system that allows for severe motor disabilities to use a computer has been

Table 1. Control subjects assessment for different parameters of the voluntary winks detection interface.

\begin{tabular}{lc}
\hline Parameter & Average score (0 to 5) \\
\hline Interface comfort & 4.06 \\
Easy handling & 4.56 \\
Order code & 4.44 \\
Delay & 3.88 \\
Test usefulness & 4.00 \\
\hline
\end{tabular}


implemented. An interface adapted to detect residual voluntary movements, eye winks, has been developed and the system uses these signals, after their processing, to control a mouse and a virtual keyboard that carry out all the tasks of these conventional peripherals. The system consists of three parts: the interface, the processing system based on the Arduino hardware platform and the software installed on the user's personal computer. This is a very low-cost technical solution and, therefore, it is available for any interested disabled person.

Taking into account that voluntary wink is an ability that disabled people can do in most cases with a similar competence to a control subject; trials were carried out, according to a specific protocol, using control subjects without the results losing validity. The system proved to be useful, the objectives were met, and it was verified that computer management was accessible to any user. In addition, an evaluation of different parameters from surveys completed by users was made. These ratings reflect the satisfaction and interest of such users in this type of rehabilitation system for people with severe motor disabilities.

As usual, any project that involves Rehabilitation Technologies is a work in progress, taking into account the views of people who have used it, until optimum performance is achieved. A greater number of trials, with both control subjects and patients interested in a daily use of the system, would be necessary to be carried out. From our experience, an adaptation of the system parameters should be carried out, essentially in order to shorten the delays on wink detection, as well as the speed of handling by patients concerning the learning curve.

\section{Acknowledgements}

This research was partially supported by the Regional Ministry of Education from Castilla y León (Spain), and by the European Social Fund.

\section{REFERENCES}

[1] A. Alonso, R. de la Rosa, A. Carrera, A. Bahillo, R. Durán and P. Fernández, "A Control System for Robots and Wheelchairs: Its Application for People with Severe Motor Disability,” In: Z. Gacovski, Ed., Mobile RobotsCurrent Trends, InTechOpen, Rijeka, 2011.

[2] A. Murai, M. Mizuguchi, M. Nishimori, T. Sayito, T. Osaka and R. Konishi, "Voice Activated Wheelchair with Collision Avoidance Using Sensor Information," Proceedings of the ICROSSICE International Joint Conference, Fukuoka, 18-21 August 2009, pp. 4232-4237.

[3] M. Teixidó, A. Guillamet, T. Pallejà, M. Tresanchez, J. Palacín, A. Fernández del Viso and C. Rebate, "Implementation of Virtual Mouse HeadMouse as a Tool to Improve ICT Accessibility," Proceedings of the IV International Congress on Design, Research Networks, and
Technology for All (DRT4all2011), Madrid, 27-29 June 2011, pp. 433-442.

[4] A. Frizera, W. Cardoso, V. Ruiz, T. FreireBastos and M. Sarcinelli, "Human-Machine Interface Based on Electrobiological Signals for Mobile Vehicles," Proceedings of the 2006 IEEE International Symposium on Industrial Electronics, Vol. 4, Montreal, 9-13 July 2006, pp. 29542959.

[5] J. del R. Millán, F. Renkens, J. Mouriño and W. Gerstner, "Noninvasive Brain-Actuated Control o a Mobile Robot by Human EEG,” IEEE Transactions on Biomedical Engineering, Vol. 51, No. 6, 2004, pp. 1026-1033. http://dx.doi.org/10.1109/TBME.2004.827086

[6] A. Alonso, R. de la Rosa, L. del Val, M. I. Jimenez and S. Franco, "A Robot Controlled by Blinking for Ambient Assisted Living,” Distributed Computing, Artificial Intelligence, Bioinformatics, Soft Computing, and Ambient Assisted Living. Lecture Notes in Computer Science, Vol. 5518, No. 1, 2009, pp. 839-842.

[7] J. M. Azkoitia, G. Eizmendi, I. Manterota, H. Zabaleta and M. Pérez, "Non-Invasive, Wireless and Universal Interface for the Control of Peripheral Devices by Means of Head Movements," Proceedings of II International Congress on Domotics, Robotics and Remote-Assistance for All (DRT4all2007), Madrid, 13-15 June 2007, pp. 211219.

[8] A. Alonso, "System Design of a Self-Guided Wheelchair for Controlled Environments," Proceedings of the Internacional Simposium on Biomechanic Methods (SIBVA '99), Valladolid, 1-3 December 1999, pp. 103-110.

[9] D. G. Evans, R. Drew and P. Blenkon, "Controlling Mouse Pointer Position Using an Infrared Head-Operated Joystick," IEEE Transactions on Rehabilitation Engineering, Vol. 8, No. 1, 2000, pp. 107-116.

http://dx.doi.org/10.1109/86.830955

[10] Y. W. Kim and J. H. Cho, “A Novel Development of Head-Set Type Computer Mouse Using Gyro Sensors for the Handicapped," Proceedings of the 2nd Annual International IEEE-EMB Special Topic Conference on Microtechnologies in Medicine \& Biology, Madison, 2-4 May 2002, pp. 356-360.

[11] X. Huo, J. Wang and M. Ghovanloo, “A Wireless TongueComputer Interface Using Stereo Differential Magnetic Field Measurement," Proceedings of the 29th Annual International Conference of the IEEE Engineering in Medicine and Biology Society, Lyon, 22-26 August 2007, pp. 5723-5726.

[12] A. Plotkin, L. Sela, A. Weissbrod, R. Kahana, L. Haviv, Y. Yeshurun, N. Soroker and N. Sobel, "Sniffing Enables Communication and Environmental Control for the Severely Disabled," Proceedings of the National Academy of Sciences of the United States of America, Vol. 107, No. 32, 2010, pp. 14413-14418.

http://dx.doi.org/10.1073/pnas.1006746107

[13] J. Fan, S. Jia, X. Li, W. Lu, J. Sheng, L. Gao and J. Yan, "Motion Control of Intelligent Wheelchair Based on Sitting Postures," Proceedings of the 2011 IEEE International Conference on Mechatronics and Automation, Beijing, 7-10 August 2011, pp. 301-306. 
http://dx.doi.org/10.1109/ICMA.2011.5985674

[14] J. M. Ford and S. J. Sheredos, "Ultrasonic Head Controller for Powered Wheelchairs," Journal of Rehabilitation Research \& Development, Vol. 32, No. 3, 1995, pp. 280284.

[15] Y. W. Kim, "Development of Headset-Type Computer Mouse Using Gyro Sensors for the Handicapped,” Electronics Letters, Vol. 38, No. 22, 2002, pp. 1313-1314. http://dx.doi.org/10.1049/el:20020950

[16] A. De Santis and D. Iacoviello, "Robust Real Time Eye Tracking for Computer Interface for Disabled People," Computer Methods and Programs in Biomedicine, Vol. 96, No. 1, 2009, pp. 1-11. http://dx.doi.org/10.1016/j.cmpb.2009.03.010

[17] M. Betke, P. Gips and P. Fleming, "The Camera Mouse: Visual Tracking of Body Features to Provide Computer
Access for People with Severe Disabilities,” IEEE Transactions on Neural Systems and Rehabilitation Engineering, Vol. 10, No. 1, 2002, pp. 1534-4320. http://dx.doi.org/10.1109/TNSRE.2002.1021581

[18] R. Barea, L. Boquete, J. M. Rodriguez-Ascariz, S. Ortega and E. López, "Sensory System for Implementing a Human-Computer Interface Based on Electrooculography," Sensors (Basel), Vol. 11, No. 1, 2011, pp. 310-328. http://dx.doi.org/10.3390/s110100310

[19] M. Yoshida, T. Itou and J. Nagata, "Development of EMG controlled mouse cursor," Proceedings of the Second Joint EMBS-BMES, 24th Annual International Conference of the Engineering in Medicine and Biology Society, Annual Fall Meeting of the Biomedical Engineering Society, Vol. 3, Houston, 23-26 October 2002, p. 2436. 